\title{
Dos casos con hallazgo citogenético de inversión del brazo largo del cromosoma 3 -inv(3q)-: reporte de casos y revisión de la literatura
}

- Luz Karime Yunis Hazbun, Jorge Cuéllar, Mike Vásquez, Karina Bautista, Victoria Torres, Juan José Yunis Londoño Servicios Médicos Yunis Turbay (Bogotá, D.C.)

Introducción: realizar la descripción de dos casos con hallazgo citogenético de inv(3q) y la revisión de la literatura sobre el valor pronóstico de este.

Materiales y métodos: se llevó a cabo estudio citogenético convencional a dos muestras de médula ósea remitidas por diagnóstico de leucemia mieloide aguda (LMA) de novo y leucemia mieloide crónica (LMC) tratada, en seguimiento en fase blástica. Se efectuó el análisis citogenético a partir de cultivo inmediato y estimulado de 72 horas.

Resultados: para el caso de la LMC, se identificó una línea celular con la presencia de inv3 y traslocación 9;22 -46,XY,inv(3)(q21q26), t(9;22)(q34;q11) [9]/46,XY [16] 1-. Para el caso de la LMA, se identificó la presencia de inv3 y monosomía del cromosoma $7-45, X X$, inv(3)(q21q26),-7 [7]/46,XX [20]-.

Conclusiones: la inv(3q) se ha reportado en LMA de novo, síndrome mielodisplásico, LMC, neoplasias mieloproliferativas y neoplasias mieloides relacionadas con la terapia. Las neoplasias mieloides con $\operatorname{inv}(3 q)$ representan un grupo de leucemias altamente resistentes a la terapia convencional con tasas de supervivencia global bajas. Este rearreglo conlleva expresión alterada de EVI1, y se ha identificado una activación de la vía RAS/RTK en un $98 \%$ de los casos. La alta incidencia de activación de esta vía brinda posibles blancos terapéuticos para terapia dirigida en este grupo de pacientes de alto riesgo. 